www.nature.com/pj

\title{
Active self-organization of microtubules in an inert chamber system
}

\begin{abstract}
Arif Md. Rashedul Kabir ${ }^{1}$, Daisuke Inoue ${ }^{2}$, Akira Kakugo ${ }^{3,4}$, Kazuki Sada $^{3}$ and Jian Ping Gong ${ }^{5}$
Microtubule-kinesin system is considered as a building block for the construction of artificial biomachines, and active selforganization of microtubules has been used to integrate their structural organization and achieve amplified functions similar to those observed in natural systems. However, the short lifetime of assembled structures has limited their use in organized systems. In the present study, we demonstrated that the use of an inert atmosphere in the self-organization of microtubules allows the assembled structures to remain active for a prolonged period of time (10 times longer). The longer lifetime achieved in the present study will facilitate the development of assembled microtubules for designing biomolecular motor-based efficient artificial biomachines with prolonged lifetimes.
\end{abstract}

Polymer Journal (2012) 44, 607-611; doi:10.1038/pj.2012.26; published online 4 April 2012

Keywords: active self-organization; biomachine; biomolecular motor; kinesin; lifetime; microtubule

\section{INTRODUCTION}

Actin-myosin and microtubule (MT)-kinesin systems are known for their fascinating in vivo activities with respect to cell motility, ${ }^{1}$ cytokinesis $^{2}$ and cellular transport. To unveil the functions of bimolecular motor systems $\mathrm{s}^{3-5}$ and to elucidate the in vivo mechanism of actin-myosin or MT-kinesin interactions, in vitro motility assay ${ }^{6,7}$ has been used over the last few decades. Currently, the in vitro motility assay is also used to determine the feasibility of constructing motor protein-based artificial biomachines, for which actin-myosin or MT-kinesin systems are considered promising building blocks. ${ }^{8-16}$ New classes of devices including nanoscale molecular shuttles, ${ }^{17,18}$ surface-imaging processes, ${ }^{19}$ force measurements ${ }^{20}$ and lab-on-a-chip devices ${ }^{21}$ have been developed using knowledge obtained through the in vitro motility assay. To improve the organizational hierarchy of motor protein-based systems with emergent functions similar to those observed in natural systems, several active self-organization (AcSO) techniques have been developed. ${ }^{22-25}$ For instance, a specific streptavidin (St)-biotin (Bt) interaction has been used in the in vitro motility assay of MTs on a kinesin-fixed surface, and a variety of well-organized MTs assemblies have been produced including linear bundles, rings and network structures, all of which showed motility comparable to that of single MT filaments. However, the lifetime of biomolecular motors is short owing to the attack of reactive oxygen species (ROS), which terminates their activity and limits the long-term utilization of assembled structures. Thus, assembled structures formed under ambient aerobic conditions remain active for only $\sim 90 \mathrm{~min}$.
To prolong the lifetime of biomolecular motors, we recently developed a device called the inert chamber system (ICS), ${ }^{26}$ which is used to perform motility assays in an oxygen-free, humid, nitrogen atmosphere. Using MT-kinesin as a model system, we showed that the lifetime of biomolecular motors could be prolonged for approximately 1 week by performing in vitro motility assays in an inert atmosphere. $^{26}$ This result may be of great importance for the construction of biomolecular motor-based devices with longer lifetimes. In the present investigation, we studied the AcSO of MTs in the ICS and found that the lifetime of assembled MTs was prolonged to almost $\sim 900 \mathrm{~min}$ (under aerobic condition, assembled MTs lost their mobility within $\sim 90 \mathrm{~min}$ ). The prolonged lifetime of organized MT structures will accelerate the development of biomolecular motor-based artificial machines with improved efficiency and longevity and widen the scope of industrial applications of MTs.

\section{EXPERIMENTAL PROCEDURES}

Preparation of tubulins and kinesin

Tubulin was purified from porcine brain using a high-concentration PIPES buffer ( $1 \mathrm{~m}$ PIPES, $20 \mathrm{~mm}$ EGTA, $10 \mathrm{~mm} \mathrm{MgCl}_{2} ; \mathrm{pH}$ adjusted to 6.8 using $\mathrm{KOH}$ ). High-molarity PIPES buffer and $80 \mathrm{~mm}$ PIPES brain reconstitution buffer (BRB80) were prepared using PIPES (Sigma, St Louis, MO, USA), and the $\mathrm{pH}$ was adjusted using $\mathrm{KOH}^{27}$ Green fluorescent protein (GFP)-fused kinesin-1 consisting of the first 560 amino acids (K560-GFP) was prepared by partially modifying the protein expression and purification methods. ${ }^{28}$

${ }^{1}$ Division of Biological Sciences, Graduate School of Science, Hokkaido University, Sapporo, Japan; ${ }^{2}$ Division of Biological Sciences, Graduate School of Life Science, Hokkaido University, Sapporo, Japan; ${ }^{3}$ Department of Chemistry, Graduate School of Science, Hokkaido University, Sapporo, Japan; ${ }^{4}$ PRESTO, Japan Science and Technology Agency (JST), Saitama, Japan and ${ }^{5}$ Faculty of Advanced Life Science, Hokkaido University, Sapporo, Japan

Correspondence: Professor A Kakugo, Department of Chemistry, Graduate School of Science, Hokkaido University Kita10, Nishi8, Kita-ku, Sapporo, Hokkaido 060-0810, Japan. E-mail: kakugo@sci.hokudai.ac.jp

Received 25 January 2012; revised 12 February 2012; accepted 14 February 2012; published online 4 April 2012 


\section{Preparation of labeled tubulins}

Rhodamine-labeled tubulins were prepared using tetramethylrhodamine succinimidyl ester (TAMRA-SE; Invitrogen, Eugene, OR, USA) according to standard techniques. ${ }^{29}$ Rhodamine-tubulin was obtained via chemical crosslinking, and the corresponding labeling ratio was equal to 1.5. The labeling ratio was determined by measuring the absorbance of the protein at $280 \mathrm{~nm}$ and determining the absorbance of tetramethylrhodamine at $555 \mathrm{~nm}$

\section{Bt labeling and stoichiometric estimation}

Bt-labeled tubulins were prepared using biotin-XX-SE (Invitrogen) according to standard techniques. ${ }^{30}$ The labeling stoichiometry was approximately 0.61 per tubulin heterodimer, as estimated by spectrometric titration using 2-(4'-hydroxyphenylazo)benzoic acid (HABA) (Wako, Osaka, Japan). ${ }^{31}$

\section{AcSO of MTs}

Bt- and rhodamine-labeled MTs were obtained by polymerizing a mixture of biotin-tubulin and rhodamine-tubulin (Bt/Tub: 0.6; final tubulin concentration: $55.6 \mu \mathrm{M})$ at $37^{\circ} \mathrm{C}$. The solution containing the MTs was diluted with motility buffer ( $80 \mathrm{~mm}$ PIPES, $1 \mathrm{~mm}$ EGTA, $2 \mathrm{~mm} \mathrm{MgCl}_{2}, 0.5 \mathrm{mg} \mathrm{ml}^{-1}$ casein, $1 \mathrm{~mm}$ DTT, $4.5 \mathrm{mg} \mathrm{ml}^{-1}$ D-glucose, $50 \mathrm{U} \mathrm{ml}^{-1}$ glucose oxidase, $50 \mathrm{U} \mathrm{ml}^{-1}$ catalase, $10 \mu \mathrm{m}$ paclitaxel, and $\sim 1 \%$ DMSO; $\mathrm{pH} 6.8$ ). In the present study, glucose, glucose oxidase and catalase were not used in all of the experiments performed in the ICS. Flow cells were prepared by placing a cover glass $\left(18 \times 18 \mathrm{~mm}^{2}\right.$; MATSUNAMI $)$ on a slide glass $\left(26 \times 76 \mathrm{~mm}^{2}\right.$; for experiments using ICS, the dimensions of the slide glass were equal to $40 \times 50 \mathrm{~mm}^{2}$ ) equipped with a pair of spacers to form a chamber with dimensions of approximately $4 \times 18 \times 0.1 \mathrm{~mm}^{3}(\sim 10 \mu \mathrm{l}) \quad(\mathrm{W} \times \mathrm{L} \times \mathrm{H})$. A single layer of Parafilm was heated to fix the spacer-separated glasses. The flow cell was filled with $0.2 \mathrm{mg} \mathrm{ml}^{-1}$ anti-GFP antibody (Invitrogen) for $15 \mathrm{~min}$ and was washed with $48 \mu \mathrm{l}$ of casein solution ( $80 \mathrm{~mm}$ PIPES, $1 \mathrm{~mm}$ EGTA, $1 \mathrm{~mm} \mathrm{MgCl}_{2}$, $\sim 0.5 \mathrm{mg} \mathrm{ml}^{-1}$ casein; $\mathrm{pH}$ adjusted to 6.8 using $\mathrm{HCl}$ ). After incubating for $5 \mathrm{~min}$ with casein solution to mask the remaining glass surface, $24 \mu \mathrm{l}$ of $100 \mathrm{nM}$ K560-GFP solution $(\sim 80 \mathrm{~mm}$ PIPES, $\sim 40 \mathrm{~mm} \mathrm{NaCl}, 1 \mathrm{~mm}$ EGTA, $1 \mathrm{~mm}$ $\mathrm{MgCl}_{2}, 0.5 \mathrm{mg} \mathrm{ml}^{-1}$ casein, $1 \mathrm{~mm}$ DTT, $4.5 \mathrm{mg} \mathrm{ml}^{-1}$ D-glucose, $50 \mathrm{U} \mathrm{ml}^{-1}$ glucose oxidase, $50 \mathrm{U} \mathrm{ml}^{-1}$ catalase, $10 \mu \mathrm{m}$ paclitaxel, $\sim 1 \%$ DMSO; pH 6.8) was introduced and incubated for $10 \mathrm{~min}$ to bind the kinesins to the antibody The flow cell was washed with $32 \mu \mathrm{l}$ of motility buffer. A dilute solution $(24 \mu \mathrm{l})$ of MTs (the concentration of MTs is provided in the results and discussion section) was introduced, incubated for $10 \mathrm{~min}$ and washed with $32 \mu \mathrm{l}$ of motility buffer. Streptavidin solution $(24 \mu \mathrm{l})$ (ST; Wako) of prescribed concentration (in motility buffer) was introduced and incubated for $10 \mathrm{~min}$, and the cell was washed with $50 \mu \mathrm{l}$ of motility buffer. Finally, AcSO was initiated by applying $24 \mu \mathrm{l}$ of ATP solution (motility buffer supplemented with $5 \mathrm{~mm}$ ATP). The time of ATP addition was set to $0 \mathrm{~h}$, and the aforementioned experiments were performed at room temperature. For AcSO in the ICS, the flow cell was fixed inside the inert chamber after the addition of ATP, as described in a previous report. ${ }^{26}$ Humid nitrogen gas was passed through the chamber to remove oxygen from chamber. Microscopic observations were obtained after passing nitrogen gas through the chamber for $1 \mathrm{~h}$, and a flow of nitrogen was applied continuously until the experiment was completed.

\footnotetext{
Microscopic images

To study the motility of the MTs, samples were illuminated with a $100 \mathrm{~W}$ mercury lamp and were visualized using epifluorescence microscopy with an oil-coupled Plan Apo $60 \times 1.40$ objective (Nikon, Melville, NY, USA). To visualize the samples, filter blocks with UV-cut specifications (TRITC: EX540/ 25, DM565, BA606/55; GFP-HQ: EX455-485, DM495, BA500-545; Nikon) were introduced into the optical path of the microscope to eliminate UV radiation and minimize its harmful effect on the samples. The images were captured using a cooled-CCD camera (Cascade II, Nippon Roper, Tokyo, Japan) connected to a PC. To capture a field of view for more than several minutes, ND (neutral density) filters (ND4, 25\% transmittance) were inserted into the illuminating light path of the fluorescence microscope to avoid photobleaching of rhodamine-labeled MTs and GFP-kinesins.
}

\section{Image analysis for motility assays}

Movies of the MT motility assays captured via fluorescence microscopy were analyzed using an image analysis software (Image Pro Plus 6.1, Media Cybernetics, Bethesda, MD, USA). MTs that moved over distances greater than $\sim 0.5 \mu \mathrm{m}$, which corresponded to 2 pixels in the digital image, were considered motile MTs. To measure the velocity of MTs, the track of MTs in six sequential images were manually detected at 10 -s intervals using the aforementioned software.

\section{RESULTS AND DISCUSSION}

The AcSO of MTs achieved using the St-Bt interaction in the in vitro motility assay is schematically shown in Figure 1. Tubulins were modified with biotin and were polymerized to form the MTs. After preparing the flow cell and immobilizing kinesins on the glass surface via anti-GFP antibodies, the MTs were applied to the flow cell and St solution of prescribed concentration was added at a specific $\mathrm{St} / \mathrm{Bt}$ ratio. Finally, ATP was added to the flow cell to initiate the selfassembly of MTs. For the AcSO of MTs in the ICS, the flow cell was fixed in the inert chamber after the addition of ATP and was incubated under an atmosphere of humid nitrogen. Using fluorescence microscopy, observations were performed after $1 \mathrm{~h}$ of incubation to decrease the oxygen concentration inside the chamber to less than 0.1 p.p.m. (parts per million). ${ }^{26}$

First, the self-organization of MTs was performed without the ICS under different conditions and various $\mathrm{St}$ to $\mathrm{Bt}$ ratios $(\mathrm{St} / \mathrm{Bt})$. Figure 2 shows the morphological changes of St- and Bt-modified MTs over time. As shown in the figure, assembly formation was observed after the addition of ATP, and the morphology of the assembled structures rapidly changed over time. Initially, bundles of MTs were preferentially formed from single filaments; however, over time, collisions among bundles of MTs led to the formation of ring-shaped structures. Prolonged observations revealed that the MT rings lost their motility within a short period of time and became stationary objects on the substrate surface. When another aliquot of ATP was added, the MT rings did not regain motility, which suggested that the observed loss of motility was not due to the depletion of ATP concentration. Rather, the loss of motility was attributed to any irreversible damage of MTs or kinesin.

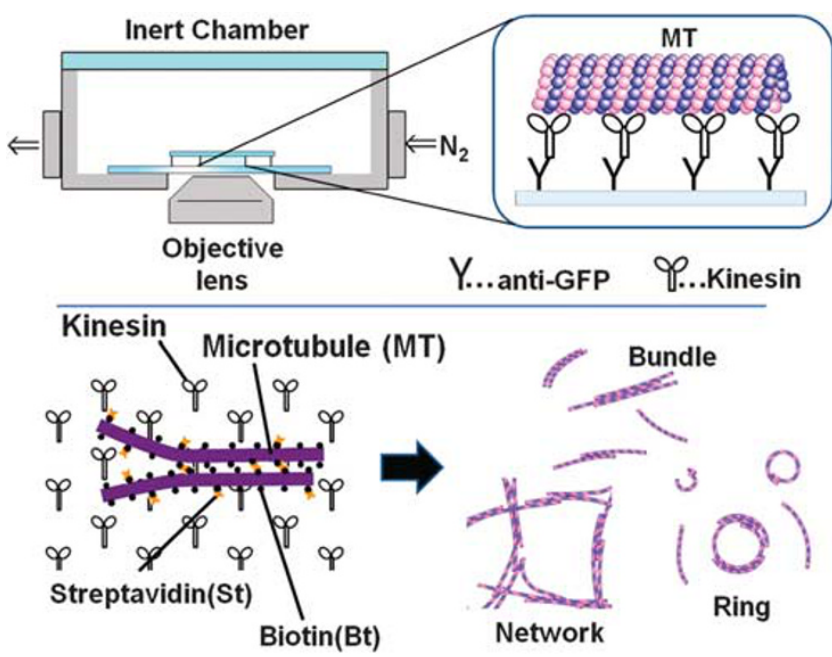

Figure 1 Schematic representation of the in vitro motility assay performed in the inert chamber system (top); active self-organization (AcSO) of microtubules (MTs) on a kinesin-coated surface due to streptavidin (St) biotin (Bt) interaction and formation of bundles, rings and network structures of MTs via AcSO (bottom). 

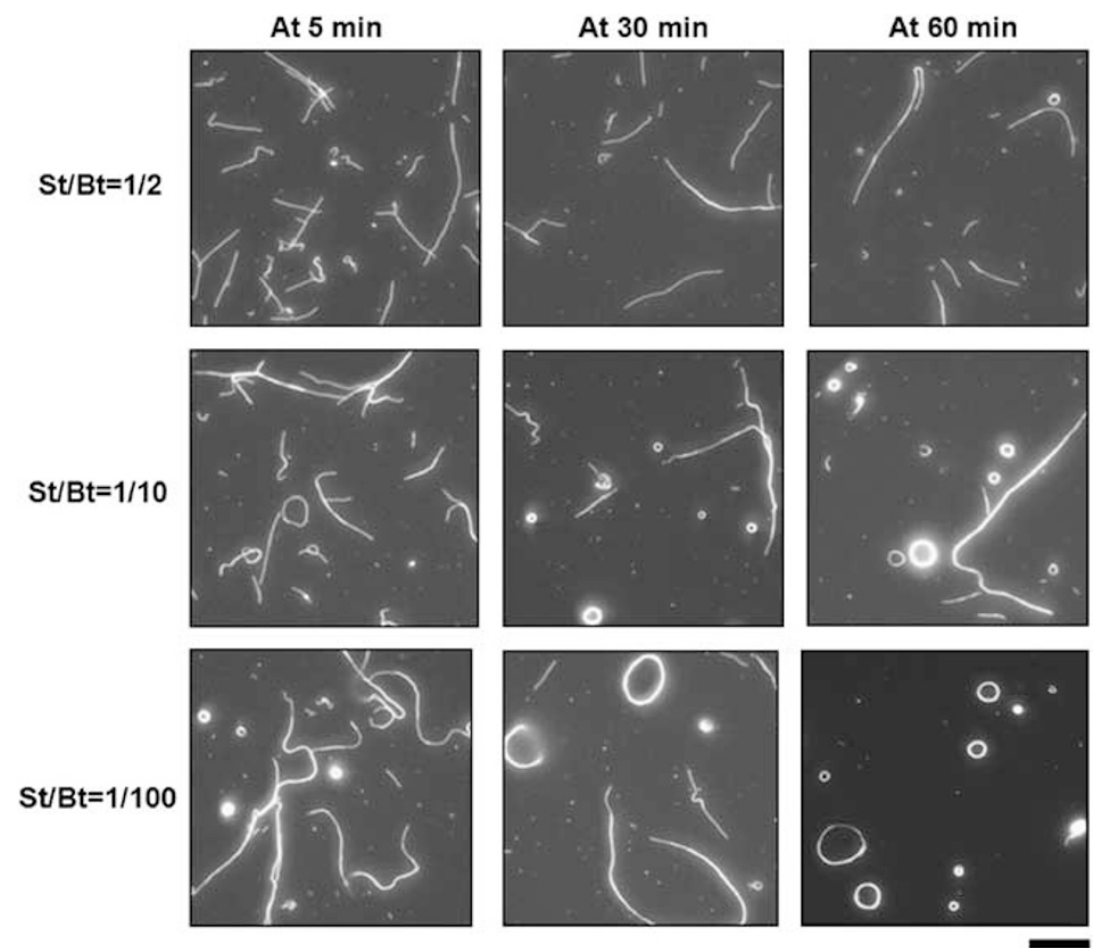

Figure 2 The effect of the streptavidin to biotin ratio (St/Bt) on the morphological changes of assembled microtubules (MTs) over time. In all three cases, the concentration of MTs was set to $111 \mathrm{~nm}$. Scale bar: $30 \mu \mathrm{m}$.

Morphological changes in the assembled MTs over time were influenced by the ratio of St/Bt used in experiments. When the St/Bt ratio was $1 / 100$, bundle formation occurred readily (within $\sim 1$ min after ATP addition), and MT rings were produced within a short period of time $(\sim 15 \mathrm{~min})$. As the $\mathrm{St} / \mathrm{Bt}$ ratio was increased to $1 / 2$, assembly formation was delayed, and bundled MTs were obtained after $20 \mathrm{~min}$. The observed delay in assembly formation was assumed to be associated with the velocity of the MTs, which was dependent on the St/Bt ratio. Hence, the effect of the St/Bt ratio on the velocity of the MTs and the time dependence of their velocity were determined (Figure 3 ). The results showed that the velocity of the MTs increased with a decrease in the $\mathrm{St} / \mathrm{Bt}$ ratio. Thus, the observed reduction in the frequency of assembly formation at the $\mathrm{St} / \mathrm{Bt}$ ratio set to $1 / 2$ may be due to the slower velocity of the MTs and a decrease in the frequency of collisions among MTs. The observed reduction in the velocity of the MTs at higher St/Bt ratio was attributed to a decrease in the driving force of kinesins due to steric hindrance with St. The motility of the assembled structures was lost within a short period of time. For instance, even when the St/Bt ratio was equal to $1 / 100$, the assembled MTs lost motility within $\sim 90 \mathrm{~min}$ after ATP addition. Moreover, when the St/Bt ratio was set to $1 / 2$, motility was lost within $45 \mathrm{~min}$. As previously discussed, the observed loss in motility was attributed to any irreversible damage of MTs or kinesin, which may be due to the attack of ROS.

Next, we optimized the concentration of MTs for assembly formation at the St/Bt ratio of $1 / 100$ and performed the AcSO of MTs in the ICS. Effective assembly formation was not observed when the concentration of MTs was as low as $11 \mathrm{~nm}$ (Figure 4). However, assembly formation was observed at a MT concentration of $111 \mathrm{nM}$. Moreover, when the MT concentration was as high as $1111 \mathrm{nM}$, a network of MTs was formed immediately after the addition of ATP. However, the network structure was subsequently transformed into entangled aggregates over time (within $\sim 30 \mathrm{~min}$ ).

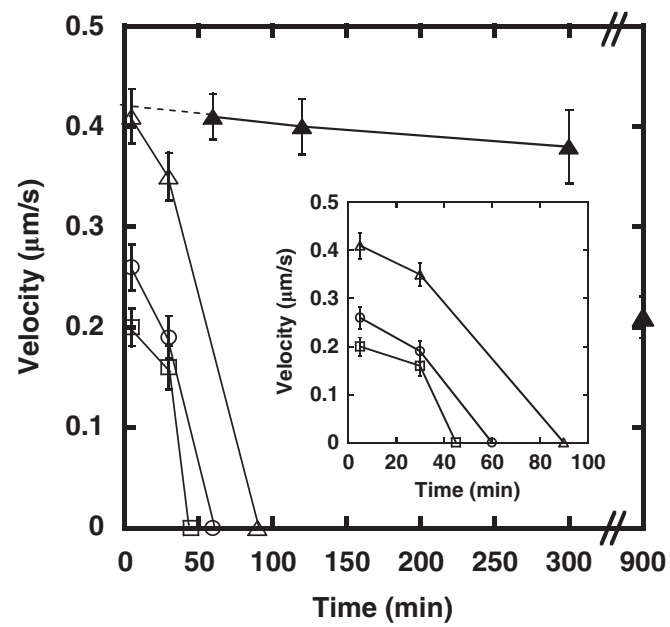

Figure 3 Change in the velocity of assembled microtubules over time under different experimental conditions. Filled and open symbols represent experiments performed with and without using the inert chamber system (ICS), respectively. The streptavidin to biotin (St/Bt) ratio was set to $1 / 100$ (triangle), 1/10 (circle) and 1/2 (square). The inset shows the results obtained without the ICS (ambient aerobic condition) in an enlarged form. For experiments performed in the ICS, the first observation was performed at $60 \mathrm{~min}$, and extrapolation of the line (dashed part) to the velocity axis represents the velocity of assembled MTs at the initial time of active selforganization.

Based on the results described above, we used the ICS to perform the AcSO of MTs at a St/Bt ratio set to $1 / 100$ and a constant MT concentration of $111 \mathrm{~nm}$. In this experiment, the first observation was performed $1 \mathrm{~h}$ after the addition of ATP because the flow cell was incubated in the chamber to remove oxygen. As shown in Figure 5, 

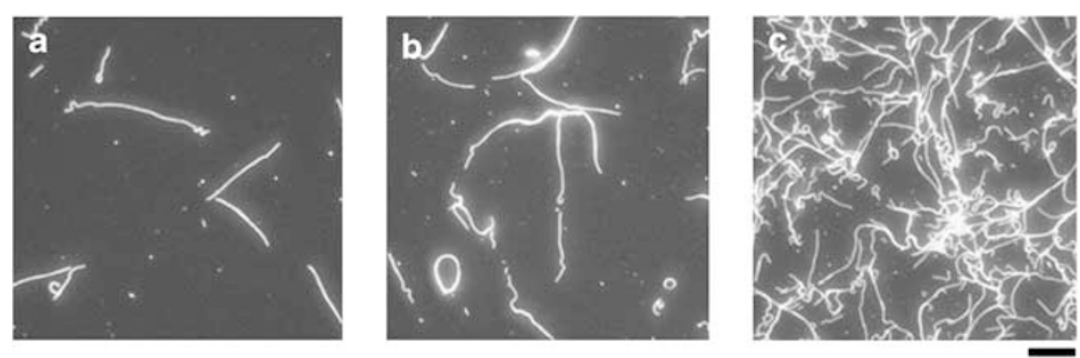

Figure 4 Effect of the concentration of microtubules (MTs) on the morphology of MT assemblies in active self-organization under ambient aerobic condition (without inert chamber system (ICS)). The concentration of MTs was set to (a) $11 \mathrm{~nm}$; (b) $111 \mathrm{~nm}$; and (c) $1111 \mathrm{~nm}$. The streptavidin to biotin (St/Bt) ratio was set to 1/100. All of the images were captured $5 \mathrm{~min}$ after ATP addition. Scale bar: $30 \mu \mathrm{m}$.

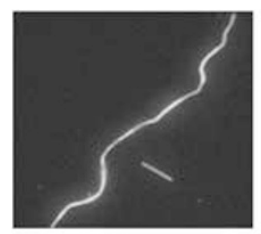

$60 \mathrm{~min}$

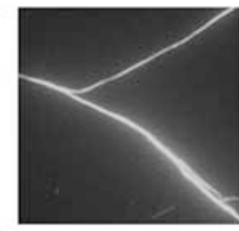

$120 \mathrm{~min}$

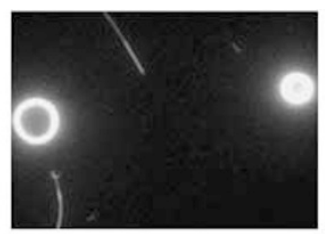

$300 \mathrm{~min}$

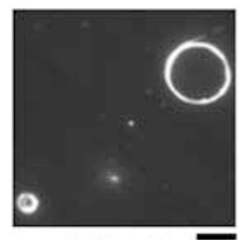

$900 \mathrm{~min}$

Figure 5 Change in the morphology of microtubule (MT) assemblies over time in the inert chamber system. The concentration of MTs used was set to $111 \mathrm{~nm}$, and the streptavidin to biotin (St/Bt) ratio was equal to 1/100. Scale bar: $30 \mu \mathrm{m}$.

the self-organization of MTs occurred in the ICS, and linear bundles of MTs were initially produced. Over time, ring-shaped MT assemblies were also formed, as observed under aerobic conditions. After $1 \mathrm{~h}$, the velocity of the assembled structures was $0.42 \mu \mathrm{m} \mathrm{s}^{-1}$, which was higher than the velocity of MT assemblies observed under aerobic conditions at the same observation time at any of the St/Bt ratios. Moreover, the assembled MT structures showed good motility, and their velocity was nearly constant for $300 \mathrm{~min}$ and gradually decreased to half of the initial velocity after $900 \mathrm{~min}$ (Figure 3). As previously reported, fluorescence microscopy can induce photochemical reactions that produce ROS in the presence of oxygen. ROS produced during sample obeservation using fluorescence microscopy can damage biomolecular motors, ${ }^{32,33}$ resulting in the loss of activity. Using the ICS, we removed oxygen from the system and demonstrated that the AcSO of MTs could be performed in an oxygen-free inert atmosphere. The ICS minimized the harmful effect of ROS on biomolecular motor protein systems, which prolonged the lifetime of the assembled MT structures.

\section{CONCLUSIONS}

In conclusion, we have demonstrated that the AcSO of MTs can be achieved in an inert nitrogen atmosphere and that assembled structures remain active for a longer time period $(\sim 900 \mathrm{~min})$ in an oxygen-free inert atmosphere. In contrast, the lifetime of the assembled structures obtained through the AcSO of MTs under aerobic condition was as short as $\sim 90 \mathrm{~min}$. The use of ICS in the self-organization of MTs has immense potential and can provide organized motor protein systems with longer lifetimes, which would aid in the development and commercialization of motor proteinbased bio-devices with high efficiencies and longer lifetimes.

\section{ACKNOWLEDGEMENTS}

This research was financially supported by the Ministry of Education, Science, Sports and Culture of Japan (Grant-in-Aid of Specially Promoted Scientific Research) and PRESTO (Japan Science and Technology Agency).
1 Cooper, J. A. The role of actin polymerization in cell motility. Annu. Rev. Physiol. 53, 585-605 (1991).

2 Umeda, M. \& Emoto, K. Membrane phospholipid dynamics during cytokinesis: regulation of actin filament assembly by redistribution of membrane surface phospholipid. Chem. Phys. Lipids 101, 81-91 (1999).

3 Harada, Y., Noguchi, A., Kishino, A. \& Yanagida, T. Sliding movement of single actin filaments on one-headed myosin filaments. Nature 326, 805-808 (1987).

4 Toyoshima, Y. Y., Kron, S. J. \& Spudich, J. A. The myosin step size: measurement of the unit displacement per ATP hydrolyzed in an in vitro assay. Proc. Natl Acad. Sci. USA 87, 7130-7134 (1990).

5 Uyeda, T. Q., Abramson, P. D. \& Spudich, J. A. The neck region of the myosin motor domain acts as a lever arm to generate movement. Proc. Natl Acad. Sci. USA 93, 4459-4464 (1996).

6 Kron, S. J., Toyoshima, Y. Y., Uyeda, T. Q. \& Spudich, J. A. Assays for actin sliding movement over myosin—coated surfaces. Methods Enzymol. 196, 399-416 (1991).

7 Kron, S. J. \& Spudich, J. A. Fluorescent actin filaments move on myosin fixed to a glass surface. Proc. Natl Acad. Sci. USA 83, 6272-6276 (1986).

8 Limberis, L. \& Stewart, R. J. Toward kinesin-powered microdevices. Nanotechnology 11, 47-51 (2000).

9 Hess, H. \& Vogel, V. Molecular shuttles based on motor proteins: active transports in synthetic environments. Rev. Mol. Biotec. 82, 67-85 (2001).

10 Böhm, K. J., Stracke, R., Mühlig, P. \& Unger, E. Motor protein-driven unidirectional transport of micrometer-sized cargoes across isopolar microtubule arrays. Nanotechnology 12, 238-245 (2001).

11 Yokokawa, R., Yoshida, Y., Takeuchi, S., Kon, T. \& Fujita, H. Unidirectional transport of a bead on a single microtubule immobilized in a submicrometre channel. Nanotechnology 17, 289-294 (2006).

12 Diehl, M. R., Zhang, K., Lee, H. J. \& Tirrell, D. A. Engineering cooperativity in biomotorprotein assemblies. Science 311, 1468-1471 (2006).

$13 \mathrm{Kim}$, T., Kao, M. T., Meyhöfer, E. \& Hasselbrink, E. F. Biomolecular motor-driven microtubule translocation in the presence of shear flow: analysis of redirection behaviours. Nanotechnology 18, 1-9 (2007).

14 Suzuki, H., Oiwa, K., Yamada, A., Sakakibara, H., Nakayama, H. \& Mashiko, S. Linear arrangement of motor protein on a mechanically deposited fluoropolymer thin film. Jpn. J. Appl. Phys. 34, 3937-3941 (1995).

15 Koten, T., Månsson, A. \& Diez, S. Towards the application of cytoskeletal motor proteins in molecular detection and diagnostic devices. Curr. Opin. Biotechnol. 21, 477-488 (2010)

16 Agarwal, A. \& Hess, H. Biomolecular motors at the intersection of nanotechnology and polymer science. Prog. Polym. Sci. 35, 252-277 (2010).

17 Kakugo, A., Sugimoto, S., Gong, J. P. \& Osada, Y. Gel machines constructed from chemically cross-linked actins and myosins. Adv. Mater. 14, 1124-1126 (2002).

18 Turner, D. C., Chang, C., Fang, K., Brandow, S. L. \& Murphy, D. B. Selective adhesion of functional microtubules to patterned silane surfaces.. Biophys. J. 69, 2782-2789 (1995).

19 Hess, H., Clemmens, J., Howard, J. \& Vogel, V. Surface imaging by self-propelled nanoscale probes. Nano Lett. 2, 113-116 (2002). 
20 Hess, H., Howard, J. \& Vogel, V. A. Piconewton forcemeter assembled from microtubules and kinesins. Nano Lett. 2, 1113-1115 (2002).

21 Martin, G. N., Heuvel, V. D. \& Dekker, C. Motor proteins at work for nanotechnology. Science 317, 333-336 (2007).

22 Kawamura, R., Kakugo, A., Osada, Y. \& Gong, J. P. Selective formation of a linearshaped bundle of microtubules. Langmuir 26, 533-537 (2010).

23 Kakugo, A., Shikinaka, K., Matsumoto, K., Gong, J. P. \& Osada, Y. Growth of large polymer-actin complexes. Bioconjugate Chem 14, 1185-1190 (2003).

24 Kwon, H. J., Tanaka, Y., Kakugo, A., Shikinaka, K., Furukawa, H., Osada, Y. \& Gong, J.P. Anisotropic nucleation growth of actin bundles: a model for determining the well-defined thickness of bundles. Biochemistry 45, 10313-10318 (2006).

25 Tamura, Y., Kawamura, R., Shikinaka, K., Kakugo, A., Osada, Y., Gong, J. P. \& Mayama, $\mathrm{H}$. Dynamic self-organization and polymorphism of microtubule assembly through active interactions with kinesin. Soft Matter 7, 5654-5659 (2011).

26 Kabir, A. M. R., Inoue, D., Kakugo, A., Kamei, A. \& Gong, J. P. Prolongation of the active lifetime of a biomolecular motor for in vitro motility assay by using an inert atmosphere. Langmuir 27, 13659-13668 (2011).
27 Castoldi, M. \& Popov, A. V. Purification of brain tubulin through two cycles of polymerization-depolymerization in a high-molarity buffer. Protein Expres. Purif 32, 83-88 (2003).

28 Case, R. B., Pierce, D. W., Nora, H. B., Cynthia, L. H. \& Vale, R. D. The directional preference of kinesin motors is specified by an element outside of the motor catalytic domain. Cell 90, 959-966 (1997).

29 Peloquin, J., Komarova, Y. \& Borisy, G. Conjugation of fluorophores to tubulin. Nat. Methods 2, 299-303 (2005).

30 Hyman, A., Drechsel, D., Kellogg, D., Salser, S., Sawin, K., Steffen, P., Wordeman, L. \& Mitchison, T. Preparation of modified tubulins. Methods Enzymol. 196, 478-485 (1991).

31 Green, N. M. Spectrophotometric determination of avidin and biotin. Methods Enzymol. 18, 418-424 (1970).

32 Dixit, R. \& Cyr, R. Cell damage and reactive oxygen species production induced by fluorescence microscopy: effect on mitosis and guidlines for non-invasive fluorescence microscopy. Plant J. 36, 280-290 (2003).

33 Guo, H., Xu, C., Liu, C., Qu, E., Yuan, M., Li, Z. \& Cheng, B. Mechanism and dynamics of breakage of fluorescent microtubules. Biophys. J. 90, 2093-2098 (2006). 